Contents List available at VOLKSON PRESS
International Conference on Education, Culture
and Social Development (ICECSD)
Journal Homepage: : https://topicsonsocialdevelop.com/

\title{
CONNECTIVES CONSTRAINTS ON CONTEXTUAL EFFECTS
}

\author{
Bai Arong \\ College of Foreign Studies, Guilin University of Technology Jiangan Road No. 12, Guilin, China. \\ *Corresponding Author Email: 261396255@qq.com
}

This is an open access article distributed under the Creative Commons Attribution License, which permits unrestricted use, distribution, and reproduction in any medium, provided the original work is properly cited

\begin{abstract}
ARTICLE DETAILS
\section{ABSTRACT}

Article History:

Received 12 November 2017 Accepted 12 December 2017 Available online 1 January 2018

According to relevance theoretic approach, there are at least three types of effects upon which new input can have on context. In this paper, I mainly observe some English discourse connectives which constrain contextual effects, for example, so, therefore, etc. derive contextual implication; moreover, after all, and, etc. strengthen the existing assumption; however, but, etc. contradict and eliminate the existing assumption. I also discuss the connectives inggiged and tegeged in Mongolian, and from the discussion we conclude that the connectives inggiged and tegeged can create a constraint on all the three types of contextual effects proposed in the relevance theoretic framework.
\end{abstract}

\section{KEYWORDS}

Relevance, connective, contextual effect

\section{INTRODUCTION}

In this paper, I will observe the three types of contextual effects in the relevance theoretic approach, and also observe some English discourse connectives which constrain on contextual effects. I will analyze and propose the connectives inggiged and tegeged in Mongolian can create a constraint on the three types of contextual effects proposed in the relevance theoretic framework.

\section{RELEVANCE AND COGNITIVE ENVIRONMENT}

Sperber and Wilson's definition of relevance first relates it to a context: Relevance:

An assumption is relevant in a context if and only if it has some contextual effect in that context [1].

Relevance is a comparative concept in some senses. Some assumptions may be more relevant than others. What is more, "The assessment of relevance, like the assessment of productivity, is a matter of balancing output against input" [1]. It does not only depend on the effect produced but also on the effort required to process it. So, they have improved on the previous definition by adopting an extent-condition format:

Extent condition 1: an assumption is relevant in a context to the extent that its contextual effects in this context are large.

Extent condition 2: an assumption is relevant in a context to the extent tha the effort required to process it in this context is small [1].

According to them, relevance is a conception about the relation between cost and benefit, which are cognitive. The cognitive benefit is the modification of the hearer's existing assumption, and the cognitive cost refers to the necessary effort for the expected modification. In other words, some assumption has a contextual effect while being processed, and can modify some element in our cognitive context. This modification can occur in the form of an erasure of some assumption from the context, a modification of the strength of some assumptions in context, or the derivation of contextual implication [1].

\section{PREVIOUS STUDIES}

\subsection{Context}

Context is a notion from the field of linguistics, and it is generally involved in its subfield, i.e. pragmatics. Human beings develop their contextual environment at all times of their life.

The conception of context has been developed and provided within the general theoretical background on human cognition. On the one hand, context is regarded as a set of features of the world, which we can express as time, place, speaker, etc., and it is treated as an objective, metaphysical state of affairs. On the other hand, it is treated as subjective, cognitive representation of the world, i.e. a set of assumptions regarding the world, which we can express as language, axioms, rules, etc.

A study state that mental processing as a highly cognitive apparatus, according to his picture of the mind [2]. When there is a stimulus that generates a need for mental processing at a cognitive level, modules are instantly activated and send signals of conceptual data to the central processing unit of the mind. Fodor's 'mental processing' can be contextual dependent if goal-oriented. When humans process a stimulus we usually do so with the view to produce an outcome, with some kind of intention.

Inspired by some researchers, claim that sometimes context will have to include all the background information, or it will be difficult to process some assumptions [3]. Sometimes, however, the relevance of information must be minimized when the effort increased without increasing the effect. In other words, an extent of context is determined by the assumption to process. Context is not given but chosen. What is given is relevance. People generally assume that the assumption they are processing is relevant, and then try to find a context in which its relevance will be maximized.

\subsection{Contextual Effect}

According to a study, there are at least the following three types of effects 
upon which new input can have on context: (i) the derivation of new assumptions as contextual implications; (ii) the strengthening of existing assumptions; (iii) the contradiction and elimination of existing assumptions [4].

Let us observe them one by one:

(i) the derivation of new assumptions as contextual implications; it occurs as conclusions are derivable from the new information and the context (e.g. old information) together. For example:

(1) a. If Bill came, the party was a success. (old information)

b. Bill came. (new information)

c. The party was a success. (contextual implication)

Suppose that the addressee has the existing assumption (1a), and if he/she is also aware of knowledge (1b), which is new information, he/she will conclude (1c).

(ii) the strengthening of existing assumptions

The strength of the assumptions is various. We may have more or less evidence for the existing assumption, or may have stronger or weaker confirmation. New information can influence the strength of the assumptions by providing further evidence or confirmation to the existing assumption.

(2) a. If Peter, Paul and Mary came to the party, it was a success.

b. Peter came to the party.

c. Paul came to the party.

d. Mary came to the party.

(3) a. If the party broke up late, it was a success.

b. The party broke up late.

(4) The party was a success.

The assumption in (2a) is old information that exists in the cognitive context already, and if we are aware of the knowledge in (2b-d), the contextual implication in (4) can be drawn out; on the other hand, the same conclusion (4) can be drawn out and strengthen on the basis of the existing assumption (3a) and the new information in (3b). That is, the new information in $(3 \mathrm{~b})$ provides further confirmation to the existing assumption (4) to strengthen it.

(iii) the contradiction and elimination of existing assumptions

When the old information and the new information contradict each other the weaker one is eliminated. Often, the old information is weaker and is eliminated; that is, the existing assumption is eliminated. See (5) below:

(5) (A saw B come out of the library holding a Russian book in his hand) A: B knows Russian.

After that, A heard B utter that 'I wish I knew Russian.' A understands as follows:

\section{(6) B does not know Russian.}

In this context, the new assumption (6) contradicts the existing assumption (5) and substitutes it, in that (6) is uttered by B himself and becomes the stronger assumption.

We saw the three types of contextual effects in the relevance theoretic approach. The new information is relevant to any context that bears cognitive effect. And the greater the cognitive effects and the smaller the effort required to derive them, the greater the relevance of the input to the individual at that time.

\section{MONGOLIAN CONNECTIVES INGGIGED, TEGEGED: CONSTRAINTS ON CONTEXTUAL EFFECTS}

A researcher discusses that discourse connectives constrain on contextual effects [5]. For example, so, therefore, etc. derive contextual implication; moreover, after all, and, etc. strengthen the existing assumption; however, but, etc. contradict and eliminate the existing assumption. See the following examples:

(7) This suggestion can be cancelled without contradiction. Therefore/so it is an implicature.

(8) A: Will you make pancakes?

B: I haven't really got time tonight. Besides there's no milk.

(9) David is here. However, you can't see him.

In (7), the proposition so/therefore introduces is a contextual implication of an assumption which has been made accessible. The researcher also claims that many of the connectives can be used to constrain the interpretation of an utterance even if they are not used to connect two segments of text [6-9]. In (8), by using besides, the speaker B provides additional evidence for an assumption which is derived from the first segment. Here, the hearer is expected to derive the conclusion that $B$ will not make pancakes from the proposition expressed by the first utterance and from the proposition expressed by the second utterance. In (9), the proposition that however introduces is inconsistent with a proposition that the speaker assumes the hearer has derived as a contextual implication the hearer can see David from the first utterance.

Now, I will demonstrate that Mongolian connectives inggiged and tegeged can create a constraint on the three types of contextual effects proposed in the relevance theoretic framework though diverse connectives also exist for each contextual effect (e.g. eimu ece, murtegen, getele) [10]. First, let us have a look at the following cases, in which inggiged and tegeged are involved in the derivation of the contextual implication:

(10) a. Batu cimadu agurlazu baina, inggiged tere cima gi bolguhu ber iregsen

Batu you to angry now so he you OBJ see to come

ugei.

not

b. Batu is angry with you now, so (inggiged) he hasn't come to see you.

(11) a. Tala yin ezi ni ebedcilezei, tegeged tere unudur hiciyel sagatazai. Tala GEN mum SUB was ill so he today class absent b. Tala's mother was ill, so (tegeged) he was absent from class today.

In (10) and (11), the preceding utterances of inggiged and tegeged are the reasons for the utterances that follow them [11]. That is, from the existing assumption that If you are angry with someone, you don't want to see $\mathrm{him} / \mathrm{her}$, and with the new information that Batu is angry with the hearer, inggiged derives the contextual implication that Batu hasn't come to see the hearer; also, tegeged derives the contextual implication that Tala was absent from class today involves the existing assumption that If Tala's Mother is ill nobody looks after her except for Tala.

Similar cases can also be seen in a dialogue or non-linguistic context. Look at the following example:

(12) (A met her boyfriend strolling with a pretty girl hand in hand in a flower garden.)

A: inggiged orcim ci minu utasu gi tushu ugei baizu side.

so recently you my call OBJ answer not have FP

(So (inggiged) you haven't answered my calls recently.)

A draws the conclusion that you haven't answered my calls recently from the context that her boyfriend has been seeing another girl, by making use of inggiged. Tegeged is also applicable here.

Now, let us observe the following situations, in which inggiged and tegeged introduce new information to strengthen the existing assumptions. On this occasion, they are sometimes used with basa together. For example:

(13) Mum: Aha cin surgaguli dagan yabuba yu? brother TOP college to go $Q$ (did your brother go to the college?)

Son: Yabugsan, tegeged torsilga hibel oroi sig harizu irene gesen. 
went and experiment if do late a little back come said

(Yes, and (tegeged) he said that if he does the experiment he would come back a little late.)

(14) A: Sagsun bumbuge nagadhu ber yabuhu yu?

basket ball play to go $Q$

(Let's go and play basketball.)

B: Bi dasgal arbin baina, inggiged basa udasgibel ezi namagi $\sharp z e h \sharp$ ber I homework many have moreover in a while mum me visit for bairi du irehu gezt baina.

lodging to com that $\mathrm{FP}$

(I have lots of homework to do, moreover/besides (inggiged basa), my mother come to visit me in a while.)

In (13), the son uses tegeged to introduce new information to increase the strength of the answer yes toward his mother's question. In (14), inggiged together with basa increases the strength of the assumption that $B$ will not go and play basketball with $A$, which is inferred by the preceding utterance that $B$ has lots of homework to do.

Finally, I will present some case in which inggiged and tegeged, on some occasions with harin, and introduce utterances that contradict the existing assumptions and eliminate them.

(15) A: Tana namadu zilugudugci bagsi tanilcagulzu \#gh gez» baigsan yum.

Tana me to adviser introduce give that said FP

(Tana said that she will introduce me to an adviser.)

B: Tegeged (harin) odo boltala anir cu ugei side.

but now by sound not FP

(But (tegeged) she hasn't contacted you yet.)

In (15), inggiged can be substituted for tegeged, and harin can be appended after each of the statements. Here, tegeged indicates that the following utterance is contradictory to the existing assumption that Tana should introduce an adviser to $A$ or denying the contextual implication that if Tana introduces you to an adviser she should have contacted you by now, and it should be eliminated.

Thus, Mongolian connectives inggiged and tegeged are involved in derivation of the contextual implications, strengthening of the existing assumptions and contradiction or elimination of the existing assumptions.

\section{SUMMARY}

I observed some English discourse connectives which constrain on the three types of contextual effects in the relevance theoretic approach in this paper. I discussed that the connectives inggiged and tegeged in Mongolian can create a constraint on the three types of contextual effects proposed in the relevance theoretic framework though diverse connectives also exist for each contextual effect (e.g. eimu ece, murtegen, getele).

\section{NOTE}

1. About the contextual effect, Refer to Sperber and Wilson $\left(1995^{2}\right)$; Carston (2002); Higashimori and Yoshimura (2003); Romero and Soria (2010); Uchida (2011).

2. SUB, OBJ, TOP, GEN, FP, Q, which are adopted in this paper, refer to subject particle, object particle, topic particle, genitive particle, final particle and question particle.

\section{ACKNOWLEDGEMENTS}

This work is supported by Guangxi University Science and Technology Research Project, project code number: KY2015LX127, “A Cross Linguistic Study: Consideration on the Boundary between Semantics and Pragmatics of the Function Words", project director: Bai Arong

\section{REFERENCES}

[1] Sperber, D., Wilson, D. 1995. Relevance: Communication \& Cognition. Cambridge: Blackwell.

[2] Fodor, J.A. 1983. The Modularity of Mind: An essay on Faculty Psychology. Cambridge: MIT Press.

[3] Blakemore, D. 1992. Understanding Utterances: An Introduction to Pragmatics. Oxford \& Cambridge: Blackwell.

[4] Carston, R. 2009. Relevance Theory: Contextualism or Pragmaticism? UCL Working Papers in Linguistics, 21, 19-26.

[5] Higashimori, I., Yoshimura, A. 2003. Kanrenseiriron no ShintenkaiNinchi to komyunike-shon. Tokyo: Kenkyuusha.

[6] Uchida, S. 2011. Goyoron no Shate:-Go kara Tekusuto e. Tokyo: Kenkyusha.

[7] Assimakopoulos, S. 2006. "Context and Relevance". Theoretical \& Applied Lingistics Postgraduate Conference. Edinburgh: University of Edinburgh.

[8] Austin, J. 1962. How to Do Things with Words. Mass: Harvard University Press.

[9] Bayantai. 1989. Өbestsen Surho Yapun Hele. Huhhot : Ubur Mongol un Surgan Humuzil un Heblel un Horiya.

[10] Blakemore, D. 1996. “Are Apposition Markers Discourse Markers?” J. Linguistics, 32, 325-347.

[11] Matsui, T. 2005. "Ninchigoyoron to Kokoro no Riron no Setsuten -Meidaitaido Rikai no Hattsu Kenkyu". Proceedings of the Pragmatics Society of Japan, 8, 105-111. 